\title{
Family of modified-contracted Schrödinger equations
}

\author{
D. R. Alcoba and C. Valdemoro \\ Instituto de Matemáticas y Física Fundamental, CSIC, Serrano 123, 28006 Madrid, Spain \\ (Received 12 January 2001; published 13 November 2001)
}

\begin{abstract}
A family of equations that combines contracted Schrödinger equations of different orders is reported here. Attention is focussed on the resulting second order, third order, and fourth order of these modified-contracted Schrödinger equations. Some of these equations are self-contained and have as fixed points those corresponding to the full-configuration interaction eigenstates. The indeterminacy, which hindered initially the use of the contracted Schrödinger equations, does not formally exist in these equations. Relations linking the lower-order reduced density matrices with the higher-order matrices are exactly incorporated into the modified-contracted Schrödinger-equations structure. The cancellation of high-order correlation terms, which is hidden in the contracted Schrödinger equations, now takes an explicit form.
\end{abstract}

DOI: 10.1103/PhysRevA.64.062105

PACS number(s): 03.65.Ge, 31.10.+z

\section{INTRODUCTION}

Since the pioneering papers of Dirac [1], Husimi [2], and Löwdin [3] an extense literature appeared on the reduced density-matrices (RDM) theory. There is a large literature on this subject that the interested reader may find in [5-7]. In particular, a detailed account of the RDMs theory can be found in Ref. [8]. In recent years a new line of research has been developed around a hierarchy of RDM equations $[4,6,9-11]$. In their matrix form, these equations can be obtained by applying a contracting mapping into the $p$-electron space to the $\mathrm{N}$-electron matrix representation of the Schrödinger equation [6]. The resulting $p$-order contracted Schrödinger equation ( $p$-CSE) [6] is equivalent to the integrodifferential equation that had previously been obtained by Cho [4], Cohen and Frishberg [9], and Nakatsuji [10]. These equations are indeterminate [12] that hindered their use until Colmenero and Valdemoro [13] proposed to approximate the high-order RDMs in terms of the lower-order RDMs $[14,15]$ and then solve the equation iteratively. A rather intense development of the theory and applications of these equations has lately taken place [16-27].

The aim of this paper is to report a family of four modified-contracted Schrödinger equations (MCSE) that broadens our understanding of the many-body problem. Here we address the many-fermion problem but the generalization for treating boson systems is straightforward. These MCSE are particular combinations of several low-order CSEs. The main property of two of these MCSEs is that they do not depend on any RDM of higher order than that of the MCSE itself; in the two other cases, the initial indeterminacy is only partly removed. In a similar way as the CSE, the MCSEs can, in principle, be solved iteratively. This question, however, still remains open since the different procedures tested have not yet achieved satisfactory convergence.

The first four sections summarize the background knowledge that is needed for developing the arguments leading to the MCSEs that are reported in Sec. V. Section VI is dedicated to discuss the MCSEs practical possibilities and the questions that still need to be investigated.

\section{BASIC DEFINITIONS}

Since the fermion RDM properties follow easily from those of the creator and annihilator operators appearing in the second-quantization definition of these matrices [25], all the developments carried out here will be expressed in this formalism. The fermion operators, $b_{i}^{\dagger}$ and $b_{i}$, will be represented in a finite basis of $2 K$ orthonormal spin orbitals and the system will have a fixed number of electrons, $N$.

\section{A. The many-body Hamiltonian}

The usual many-body Hamiltonian is

$$
\hat{H}=\sum_{i, p} \varepsilon_{i p} b_{i}^{\dagger} b_{p}+\frac{1}{2} \sum_{i, k, p, q}\langle i k \mid p q\rangle b_{i}^{\dagger} b_{k}^{\dagger} b_{q} b_{p} .
$$

This Hamiltonian may be exactly rewritten as

$$
\hat{H}=\sum_{i, k, p, q}{ }^{0} H_{i k ; p q} b_{i}^{\dagger} b_{k}^{\dagger} b_{q} b_{p}
$$

where ${ }^{0} \mathrm{H}$ has the form

$$
{ }^{0} H_{i k, p q}=\frac{1}{2}\left(\frac{\varepsilon_{i p} \delta_{k q}+\varepsilon_{k q} \delta_{i p}}{N-1}+\langle i k \mid p q\rangle\right),
$$

where $\varepsilon$ and $\langle i k \mid p q\rangle$ are the usual one- and two-electron integrals, respectively (this latter in the Condon and Shortley notation).

\section{B. The $p$-order RDM}

The general $p$-RDM second-quantization definition that will be used here is

$$
{ }^{p} D_{i_{1} i_{2} \cdots i_{p} ; j_{1} j_{2} \cdots j_{p}}^{\Phi \Phi^{\prime}}=\frac{1}{p !}\left\langle\Phi\left|b_{i_{1}}^{\dagger} b_{i_{2}}^{\dagger} \cdots b_{i_{p}}^{\dagger} b_{j_{p}} \cdots b_{j_{2}} b_{j_{1}}\right| \Phi^{\prime}\right\rangle .
$$

Relation (4) is the definition of the $p$-order transition reduced density matrix ( $p$-TRDM) when $\Phi \neq \Phi^{\prime}$. In what follows, unless they are needed, the $\Phi$ superscripts will be omitted. 
The lower-order RDMs are related to the higher-order RDMs through the action of a contracting mapping [6].

\section{THE CONTRACTED SCHRÖDINGER EQUATIONS}

When contracting the matrix form of the Schrödinger equation into the one-electron space, one obtains $[6,17]$

$$
\left\langle\Phi\left|\hat{H} b_{p}^{\dagger} b_{r}\right| \Phi\right\rangle=E\left\langle\Phi\left|b_{p}^{\dagger} b_{r}\right| \Phi\right\rangle \equiv E^{1} D_{p ; r},
$$

where $\hat{H}$ is the $N$-body Hamiltonian operator just described, $E$ is the energy, and $\Phi$ its corresponding eigenstate. When replacing the $\hat{H}$ by its explicit formula (2) and transforming the left-hand side (lhs) into its normal form, one obtains the 1-CSE

$$
\begin{aligned}
E{ }^{1} D_{p ; q} & =22 ! \sum_{j}\left({ }^{0} H^{2} D\right)_{p j ; q j}+3 ! \sum_{i, j, k, l}{ }^{0} H_{i j ; k l}{ }^{s} D_{p i j ; q k l} \\
& \equiv \mathcal{M}_{p ; q} .
\end{aligned}
$$

In what follows ${ }^{p} \mathcal{M}$ will denote the $p$-order matrix formed by the right-hand side (rhs) of the $p$-CSE.

The 2-CSE is obtained in a similar way by contracting the matrix form of the Schrödinger equation from an $N$-electron representation to a two-electron representation. This matrix has the form

$$
\begin{aligned}
2 ! E{ }^{2} D_{p q ; r s}= & 2 ! 2\left({ }^{0} H^{2} D\right)_{p q ; r s}+3 ! 2 \sum_{i, k, l}\left({ }^{0} H_{q l ; k l}{ }^{3} D_{r s i ; p k l}\right. \\
& \left.+{ }^{0} H_{i p ; k l}{ }^{3} D_{r s i ; l q k}\right) \\
& +4 ! \sum_{i, j, k, l}{ }^{0} H_{i j ; k l}{ }^{4} D_{r s i j ; p q k l} \equiv{ }^{2} \mathcal{M}_{p q ; r s} .
\end{aligned}
$$

As can be seen the 1-CSE depends not only on the 1-RDM but also on the 2-and the 3-RDM. Similarly, the 2-CSE depends not only on the 2-RDM but also on the 3and 4-RDMs. This fact lies at the root of the indeterminacy of these equations. As already mentioned, in the method proposed by Colmenero and Valdemoro [13] a set of algorithms for approximating the higher-order RDMs in terms of the lower-order RDMs [14,15] allows these equations to be solved iteratively.

Although the 1-CSE is the most attractive one, its usefulness is limited by the fact that it can be exactly satisfied not only by the eigenstate RDMs but also by those RDMs corresponding to the Hartree-Fock solution. In fact, in all our attempts to solve this equation the convergence has always tended towards the Hartree-Fock solution.

\section{THE CORRELATION MATRICES}

A $p$-RDM element (for $p>1$ ) may be decomposed into different terms involving elements of lower-order RDMs and elements of a different kind of matrices that describe correlation effects. Thus, with the use of straightforward secondquantization algebra $[18,26]$ one finds

$$
\begin{aligned}
2 !{ }^{2} D_{i j ; m l}= & { }^{1} D_{i ; m}{ }^{1} D_{j ; l}-{ }^{1} D_{i ; l} \delta_{j ; m}+{ }^{2} C_{i j ; m l}, \\
3 !{ }^{3} D_{i k m ; j l n}= & -2 !{ }^{2} D_{i k ; j n} \delta_{m l}+2 !{ }^{2} D_{i k ; l n} \delta_{j m} \\
& +2 !{ }^{2} D_{i k ; j l}{ }^{1} D_{m ; n}+{ }^{3 ; 2,1} C_{i k m ; j l n},
\end{aligned}
$$

and

$$
\begin{aligned}
4 !{ }^{4} D_{i j k l ; p g r s}= & 2 !{ }^{2} D_{i j ; r s}\left(\delta_{q k} \delta_{p l}-\delta_{p k} \delta_{q l}\right)+3 !{ }^{3} D_{i j l ; q r s} \delta_{k p} \\
& +3 !{ }^{3} D_{i j k ; p r s} \delta_{l q}-3 !{ }^{3} D_{i j l ; p r s} \delta_{k ; q} \\
& -3 !{ }^{3} D_{i j k ; q r s} \delta_{l ; p}+2 !{ }^{2} D_{i j ; p q} 2 !{ }^{2} D_{k l ; r s} \\
& +4 ; 2,2 C_{i j k l ; p q r a},
\end{aligned}
$$

where the matrices ${ }^{p} C$ are $p$-order correlation matrices $[19,25,26]$ that involve products of TRDM elements. Calling $\Phi$ the eigenstate that is being considered and $\Phi^{\prime}$ all the other eigenstates of the system's spectrum the correlation matrices appearing in relations (8), (9), and (10) have the following structures

$$
\begin{aligned}
{ }^{2} C_{i j ; p q} & =\sum_{\phi^{\prime} \neq \phi}\left\langle\Phi\left|b_{i}^{\dagger} b_{p}\right| \Phi^{\prime}\right\rangle\left\langle\Phi^{\prime}\left|b_{j}^{\dagger} b_{q}\right| \Phi\right\rangle \\
& \equiv \sum_{\Phi^{\prime} \neq \Phi}{ }^{1} D_{i ; p}^{\Phi \Phi^{\prime}}{ }^{1} D_{j ; q}^{\Phi^{\prime} \Phi}
\end{aligned}
$$

that describes the pure two-body correlation effects,

$$
{ }^{(3 ; 2,1)} C_{i j k ; p q r}=2 ! \sum_{\Phi^{\prime} \neq \Phi}{ }^{2} D_{i j ; p q}^{\Phi \Phi^{\prime}}{ }^{1} D_{k ; r}^{\Phi^{\prime} \Phi},
$$

which describes a combination of pure two- and three-body correlation effects. Thus ${ }^{(3 ; 2,1)} C$ may be decomposed into three terms involving two ${ }^{2} C$ elements and a ${ }^{(3 ; 1,1,1)} C$ element,

$$
{ }^{(4 ; 2,2)} C_{i j k l ; p q r s}=2 ! 2 ! \sum_{\Phi^{\prime} \neq \Phi}{ }^{2} D_{i j ; p q}^{\Phi \Phi^{\prime}}{ }^{2} D_{k l ; r s}^{\Phi^{\prime} \Phi},
$$

which describes a combination of pure two-, three-, and fourbody correlation effects since the ${ }^{(4 ; 2,2)} C$ element may be decomposed into a combination of ${ }^{2} C,{ }^{(3 ; 1,1,1)} C$, and ${ }^{(4 ; 1,1,1,1)} C$ elements.

In general, the symbol ${ }^{(p ; 2, x, y)} C$ denotes that the $C$ is a $p$-order correlation matrix involving (from left to right) a product of elements of a 2-TRDM, and $x$-TRDM, and a $y$-TRDM.

All these matrices can be understood as describing global correlation effects due to virtual excitations and deexcitations of two, three, . ., p-electrons. It should be noted that they cannot be decomposed into terms that depend only on RDM elements.

\section{THE MODIFIED-CONTRACTED SCHRÖDINGER EQUATIONS}

A set of relations linking $C$ matrices of different orders $(C$ relations) have recently been reported [27]. These $C$ relations 
establish the set of necessary conditions that the correlation matrices must fulfill when they correspond to eigenstates of the Hamiltonian. These conditions imply that the action of matrix ${ }^{0} \mathrm{H}$ on the 2- $\mathrm{C}$ matrices cancels out the action of this same ${ }^{0} \mathrm{H}$ matrix on the 3- and 4- $\mathrm{C}$ matrices. The reason that leads to the $C$ relations given in [27] can also lead to the general result

$$
\sum_{i j k l}{ }^{0} H_{i j ; k l}{ }^{(p, 2, x, y, \cdots)} C_{i j v_{1} \cdots v_{x} t_{1} \cdots t_{y} \cdots ; k l \omega_{1} \cdots \omega_{x} z_{1} \cdots z_{y} \cdots}=0,
$$

where $p=2+x+y+\cdots$. That is, irrespective of the exact structure of the $p-C$ matrices, a sum of products of this type will vanish. As will be shown, these vanishing terms appear in the deduction of the MCSEs. It is easy to show that when the $C$ matrices involved are of the type ${ }^{(p ; 2, x)} C$ with $x>1$ these relations are also sufficient conditions to guarantee that these matrices correspond to eigenstates of the Hamiltonian.

\section{A. The second-order modified-contracted Schrödinger equation}

Let us consider again the 2-CSE

$$
{ }^{2} \mathcal{M}_{p q ; r s}=\left\langle\Phi\left|\hat{H} b_{p}^{\dagger} b_{q}^{\dagger} b_{s} b_{r}\right| \Phi\right\rangle=E 2 !{ }^{2} D_{p q ; r s},
$$

and let us rearrange the operators of the lhs as

$$
\begin{aligned}
\left\langle\Phi\left|\hat{H} b_{p}^{\dagger} b_{q}^{\dagger} b_{s} b_{r}\right| \Phi\right\rangle= & -\delta_{r q}\left\langle\Phi\left|\dot{H} b_{p}^{\dagger} b_{s}\right| \Phi\right\rangle \\
& +\left\langle\Phi\left|\hat{H} b_{p}^{\dagger} b_{r} b_{q}^{\dagger} b_{s}\right| \Phi\right\rangle .
\end{aligned}
$$

Inserting twice the unit operator

$$
\hat{I}=|\Phi\rangle\left\langle\Phi\left|+\sum_{\Phi^{\prime} \neq \Phi}\right| \Phi^{\prime}\right\rangle\left\langle\Phi^{\prime}|\equiv| \Phi\right\rangle\langle\Phi|+\hat{Q}
$$

in the last term of Eq. (16)

$$
\left\langle\Phi\left|\hat{H} \hat{I} b_{p}^{\dagger} b_{r} \hat{I} b_{q}^{\dagger} b_{s}\right| \Phi\right\rangle,
$$

Eq. (16) becomes

$$
\begin{aligned}
{ }^{2} \mathcal{M}_{p q ; r s}= & \left\langle\Phi\left|\hat{H} b_{p}^{\dagger} b_{q}^{\dagger} b_{s} b_{r}\right| \Phi\right\rangle=-\delta_{r q}{ }^{1} \mathcal{M}_{p ; s}+{ }^{1} \mathcal{M}_{p ; r}{ }^{1} D_{q ; s} \\
& +E^{2} C_{p q ; r s}+{ }^{(4 ; 2,1,1)} 0_{p q ; r s}
\end{aligned}
$$

where

$$
{ }^{(4 ; 2,1,1)} 0_{p q ; r s}=\sum_{i j k l}{ }^{0} H_{i j ; k l}{ }^{(4 ; 2,1,1)} C_{i j p q ; k l r s} .
$$

Because of relation (14), this term vanishes when the equation is satisfied. The labels of the zero symbol appearing in the lhs of Eq. (20) coincide with those of the $C$ matrix involved in the relation after omitting the indices over which the sum is carried out.

Equation (16) may therefore be written as

$$
E 2 !{ }^{2} D_{p q ; r s}=-\delta_{r q}{ }^{1} \mathcal{M}_{p ; s}+{ }^{1} \mathcal{M}_{p ; r}{ }^{1} D_{q ; s}+E^{2} C_{p q ; r s}
$$

This result gives a second-order MCSE formally equivalent to the 2-CSE but which does not depend explicitly on the 4-RDM.

Already at this stage two-important questions arise. To what extent the vanishing terms can be ignored? and how to impose the constraints on the correlation matrices implied by them? At present these questions remain open. In order to take into account the vanishing terms it is useful to combine the previous development with another alternative transformation of Eq. (15). It consists in inserting the development of the unity after the Hamiltonian operator that gives

$$
2 ! E^{2} D_{p q ; r s}+{ }^{(4 ; 2,2)} 0_{p q ; r s}={ }^{2} \mathcal{M}_{p q ; r s} .
$$

Note, that the condition ${ }^{(4 ; 2,2)} 0=0$ is therefore equivalent to the 2-CSE. Combining now Eq. (22) with Eq. (19) one has

$$
\begin{aligned}
E^{2} D_{p q ; r s}= & -\delta_{r q}{ }^{1} \mathcal{M}_{p ; s}+{ }^{1} \mathcal{M}_{p ; r}{ }^{1} D_{q ; s}+E^{2} C_{p q ; r s} \\
& +\delta_{r q}{ }^{(3 ; 2,1)} 0_{p ; s}-{ }^{1} D_{q ; s}{ }^{(3 ; 2,1)} 0_{p ; r},
\end{aligned}
$$

where the relation

$$
{ }^{4 ; 2,2} 0_{p q ; r s}=-\delta_{r q}{ }^{(3 ; 2,1)} 0_{p ; s}+{ }^{(3 ; 2,1)} O_{p ; r}{ }^{1} D_{q ; s}{ }^{(4 ; 2,1,1)} 0_{p q ; r s} .
$$

has been used. Equations (21) and (23) show that the original indeterminacy of the 1-CSE has been partially removed.

\section{B. The third-order modified-contracted Schrödinger equation}

Proceeding as in the previous paragraph, the starting relation is

$$
{ }^{3} \mathcal{M}_{i k m ; j l n}=\left\langle\Phi\left|\hat{H} b_{i}^{\dagger} b_{k}^{\dagger} b_{m}^{\dagger} b_{n} b_{l} b_{j}\right| \Phi\right\rangle=E 3 !{ }^{3} D_{i k m ; j l n} .
$$

Rearranging the order of the operators and using the unit operator (17)

$$
\begin{aligned}
E 3 !{ }^{3} D_{i k m ; j l n}= & \delta_{m j}{ }^{2} \mathcal{M}_{i k ; l n}-\delta_{m l}{ }^{2} \mathcal{M}_{i k ; j n} \\
& +\left\langle\Phi\left|\hat{H} b_{i}^{\dagger} b_{k}^{\dagger} b_{l} b_{j} b_{m}^{\dagger} b_{n}\right| \Phi\right\rangle .
\end{aligned}
$$

Let us now transform the last term of this equation by inserting repeatedly the unit operator

$$
\begin{aligned}
\left\langle\Phi\left|\hat{H} b_{i}^{\dagger} b_{k}^{\dagger} b_{l} b_{j} \hat{I} b_{m}^{\dagger} b_{n}\right| \Phi\right\rangle= & { }^{1} D_{m ; n}{ }^{2} \mathcal{M}_{i k ; j l} \\
& +\left\langle\Phi\left|\hat{H} b_{i}^{\dagger} b_{k}^{\dagger} b_{l} b_{j} \hat{Q} b_{m}^{\dagger} b_{n}\right| \Phi\right\rangle \\
= & { }^{1} D_{m ; n}{ }^{2} \mathcal{M}_{i k ; j l} \\
& +\left\langle\Phi\left|\hat{H} \hat{I} b_{i}^{\dagger} b_{k}^{\dagger} b_{l} b_{j} \hat{Q} b_{m}^{\dagger} b_{n}\right| \Phi\right\rangle \\
= & { }^{1} D_{m ; n}{ }^{2} \mathcal{M}_{i k ; j l}+E{ }^{(3 ; 2,1)} C_{i k m ; j l n} \\
& +{ }^{(5 ; 2,2,1)} 0_{i k m ; j l n}
\end{aligned}
$$

the equation thus becomes 


$$
\begin{aligned}
E 3 !{ }^{3} D_{i k m ; j l n}= & \delta_{m j}{ }^{2} \mathcal{M}_{i k ; l n}-\delta_{m l}{ }^{2} \mathcal{M}_{i k ; j n}+{ }^{2} \mathcal{M}_{i k ; j l}{ }^{1} D_{m ; n} \\
& +E^{(3 ; 2,1)} C_{i k m ; j l n}+{ }^{(5 ; 2,2,1)} 0_{i k m ; j l n}
\end{aligned}
$$

and, since the ${ }^{(5 ; 2,2,1)} 0_{i k m ; j l n}$ term disappears when the equation is satisfied it may formally be omitted. Thus,

$$
\begin{aligned}
E 3 !{ }^{3} D_{i k m ; j l n}= & \delta_{m j}{ }^{2} \mathcal{M}_{i k ; l n}-\delta_{m l}{ }^{2} \mathcal{M}_{i k ; j n}+{ }^{2} \mathcal{M}_{i k ; j l}{ }^{1} D_{m ; n} \\
& +E^{(3 ; 2,1)} C_{i k m ; j l n} .
\end{aligned}
$$

Similarly to Eq. (21), this equation shows that the original indeterminacy of the 2-CSE has been partially removed. On the other hand, this 3-MCSE takes a self-contained form when the matrix ${ }^{2} \mathcal{M}$ is replaced according to Eq. (19) and the vanishing terms are neglected.

$$
\begin{aligned}
E 3 !{ }^{3} D_{i k m ; j l n}= & \left(\delta_{m l} \delta_{k j}-\delta_{m j} \delta_{k l}\right)^{1} \mathcal{M}_{i ; n} \\
& +\left({ }^{1} D_{m ; n}{ }^{1} D_{k ; l}-\delta_{m l}{ }^{1} D_{k ; n}\right)^{1} \mathcal{M}_{i ; j} \\
& +\left(\delta_{m j}{ }^{1} D_{k ; n}-\delta_{k j}{ }^{1} D_{m ; n}\right)^{1} \mathcal{M}_{i ; l} \\
& +E \delta_{m j}{ }^{2} C_{i k ; l n}-E \delta_{m l}{ }^{2} C_{i k ; j n} \\
& +E{ }^{1} D_{m ; n}{ }^{2} C_{i k ; j l}+E{ }^{(3 ; 2,1)} C_{i k m ; j l n}
\end{aligned}
$$

As in the 2-MCSE case, an alternative expression from which the higher-order vanishing terms have been canceled out, can be obtained. Thus, when the unit operator is inserted in relation (25) after the Hamiltonian operator, one has

$$
\begin{aligned}
E 3 !{ }^{3} D_{i k m ; j l n}+{ }^{(5 ; 2,3)} 0_{i k m ; j l n} & =\left\langle\Phi\left|\hat{H} b_{i}^{\dagger} b_{k}^{\dagger} b_{m}^{\dagger} b_{n} b_{l} b_{j}\right| \Phi\right\rangle \\
& ={ }^{3} \mathcal{M}_{i k m ; j l n} .
\end{aligned}
$$

This relation can be combined with Eq. (28) giving

$$
\begin{aligned}
E 3 !{ }^{3} D_{i k m ; j l n}= & \delta_{m j}\left({ }^{2} \mathcal{M}_{i k ; l n}-{ }^{(4 ; 2,2)} 0_{i k ; l n}\right) \\
& -\delta_{m l}\left({ }^{2} \mathcal{M}_{i k ; j n}-{ }^{(4 ; 2,2)} 0_{i j ; j n}\right) \\
& +{ }^{1} D_{m ; n}\left({ }^{2} \mathcal{M}_{i k ; j l}-{ }^{(4 ; 2,2)} 0_{i k ; j l}\right) \\
& +E^{(3 ; 2,1)} C_{i k m ; j l n} .
\end{aligned}
$$

That is, after decomposing to the outmost the fifth-order vanishing terms, only the ${ }^{(4 ; 2,2)} 0$ terms remain. Again, when using Eqs. (19) and (24) a self-contained equation is obtained

$$
\begin{aligned}
E 3 !{ }^{3} D_{i k m ; j l n}= & \left(\delta_{m l} \delta_{k j}-\delta_{m j} \delta_{k l}\right)\left({ }^{1} \mathcal{M}_{i ; n}-{ }^{(3 ; 2,1)} 0_{i ; n}\right) \\
& +\left({ }^{1} D_{m ; n}{ }^{1} D_{k ; l}-\delta_{m l}{ }^{1} D_{k ; n}\right) \\
& \times\left({ }^{1} \mathcal{M}_{i ; j}-{ }^{(3 ; 2,1)} 0_{i ; j}\right) \\
& +\left(\delta_{m j}{ }^{1} D_{k ; n}-\delta_{k j}{ }^{1} D_{m ; n}\right) \\
& \times\left({ }^{1} \mathcal{M}_{i ; l}-{ }^{(3 ; 2,1)} 0_{i ; l}\right)+E \delta_{m j}{ }^{2} C_{i k ; l n} \\
& -E \delta_{m l}{ }^{2} C_{i k ; j n}+E{ }^{1} D_{m ; n}{ }^{2} C_{i k ; j l} \\
& +E{ }^{(3 ; 2,1)} C_{i k m ; j l n} .
\end{aligned}
$$

Note that this last equation is not only self-contained because the highest RDM's order is three on both sides of the equation but also because the higher-order vanishing terms have been canceled out. Although this is a very appealing equation, it should be recalled that the ${ }^{1} \mathcal{M}$ (1-CSE) is not only satisfied by the full-configuration interaction set of RDMs but also by the Hartree-Fock RDMs, which limits its usefulness.

\section{The fourth-order modified-contracted Schrödinger equation}

Since the arguments needed for obtaining the 4-MCSE are similar to those used in the two previous paragraphs only the final results are included here. Thus, the 4-MCSE equation is

$$
\begin{aligned}
E 4 !{ }^{4} D_{i j k l ; p q r s}= & \left(\delta_{k q} \delta_{l p}-\delta_{l q} \delta_{k p}\right)^{2} \mathcal{M}_{i j ; r s}+\delta_{l q}{ }^{3} \mathcal{M}_{i j k ; p r s}+\delta_{k p}{ }^{3} \mathcal{M}_{i j l ; q r s}-\delta_{l p}{ }^{3} \mathcal{M}_{i j k ; q r s}-\delta_{k q}{ }^{3} \mathcal{M}_{i j l ; p r s} \\
& +{ }^{2} \mathcal{M}_{i j ; p q} 2 !^{2} D_{k l ; r s}+E \\
\equiv & \left(\delta_{l q} \delta_{k p}-\delta_{k q} \delta_{l p}\right)^{2} \mathcal{M}_{i j ; r s}+\left(\delta_{k q} \delta_{l j}-\delta_{l q} \delta_{k r}\right)^{2} \mathcal{M}_{i j ; p s}+\left(\delta_{l p} \delta_{k r}-\delta_{k p} \delta_{l r}\right)^{2} \mathcal{M}_{i j ; q s} \\
& +\left(\delta_{l q}{ }^{1} D_{s ; k}-\delta_{k q}{ }^{1} D_{s ; l}\right)^{2} \mathcal{M}_{i j ; p r}+\left(\delta_{k p}{ }^{1} D_{l ; s}-\delta_{l p}{ }^{1} D_{k s}\right)^{2} \mathcal{M}_{i j ; q r}+2 !^{2} D_{k l ; r s}{ }^{2} \mathcal{M}_{i j ; p q} \\
& +\delta_{l q} E^{(3 ; 2,1)} C_{i j k ; p r s}+\delta_{k p} E^{3 ; 2,1} C_{i j l ; q r s}-\delta_{k q} E^{(3 ; 2,1)} C_{i j l ; p r s}-\delta_{l p} E^{(3 ; 2,1)} C_{i j k ; q r s}+E^{(4 ; 2,2)} C_{i j k l ; p q r s}
\end{aligned}
$$

and the alternative equation where the vanishing terms appear explicitly and where only the ${ }^{2} \mathcal{M}$ (the 2-CSE) appears is

$$
\begin{aligned}
E 4 !{ }^{4} D_{i j k l ; p q r s}= & \left(\delta_{l q} \delta_{k p}-\delta_{k q} \delta_{l p}\right)\left({ }^{2} \mathcal{M}_{i j ; r s}-{ }^{(4 ; 2,2)} 0_{i j ; r s}\right)+\left(\delta_{k q} \delta_{l r}-\delta_{l q} \delta_{k r}\right)\left({ }^{2} \mathcal{M}_{i j ; p s}-{ }^{(4 ; 2,2)} 0_{i j ; p s}\right)+\left(\delta_{l p} \delta_{k r}-\delta_{k p} \delta_{l r}\right) \\
& \times\left({ }^{2} \mathcal{M}_{i j ; q s}-{ }^{(4 ; 2,2)} 0_{i j ; q s}\right)+\left(\delta_{l q}{ }^{1} D_{s ; k}-\delta_{k q}{ }^{1} D_{s ; l}\right)\left({ }^{2} \mathcal{M}_{i j ; p r}-{ }^{(4 ; 2,2)} 0_{i j ; p r}\right)+\left(\delta_{k p}{ }^{1} D_{l ; s}-\delta_{l p}{ }^{1} D_{k ; s}\right) \\
& \left.\times\left({ }^{2} \mathcal{M}_{i j ; q r}-{ }^{(4 ; 2,2)} 0_{i j ; q r}\right)+2 !{ }^{2} D_{k l ; r s}{ }^{2} \mathcal{M}_{i j ; p q}-{ }^{(4 ; 2,2)} 0_{i j ; p q}\right)+\delta_{l q} E{ }^{(3: 3,1)} C_{i j k ; p r s}+\delta_{k p} E{ }^{(3 ; 2,1)} C_{i j l ; q r s} \\
& -\delta_{k q} E^{(3 ; 2,1)} C_{i j l ; p r s}-\delta_{l p} E^{(3 ; 2,1)} C_{i j k ; q r s}+E^{(4 ; 2,2)} C_{i j k l ; p q r s .} .
\end{aligned}
$$


This last equation is not only self-contained because the highest RDM's order is four on both sides of the equation but also because the higher-order vanishing terms have been canceled out.

Although these relations are exact it should be noted that they do not have the RDM's antisymmetry property built in. This point will be discussed in some detail in the last section.

\section{Relation between the modified-contracted Schrödinger equations's structure and the reduced density-matrices decomposition formulas}

It is interesting to note that there is a short-cut method for deducing the MCSEs. Thus, let us, for instance, consider the 2-RDM decomposition formula (8) and let us multiply it by the energy $E$

$$
E 2 !{ }^{2} D_{i j ; m l}=E{ }^{1} D_{i ; m}{ }^{1} D_{j ; l}-E{ }^{1} D_{i ; l} \delta_{j ; m}+E{ }^{2} C_{i j ; m l},
$$

then replacing $E{ }^{1} D$ by ${ }^{1} \mathcal{M}$ one has

$$
E 2 !{ }^{2} D_{i j ; m l}={ }^{1} \mathcal{M}_{i ; m}{ }^{1} D_{j ; l}-{ }^{1} \mathcal{M}_{i ; l} \delta_{j ; m}+E{ }^{2} C_{i j ; m l},
$$

that is, the 2-MCSE is reobtained. Similar short-cut deductions can be written for the 3-and 4-MCSEs. By deducing the MCSEs in this way, the ${ }^{p} 0$ vanishing correlation terms do not appear. As will be discussed in the last section, apart from their theoretical interest, these ${ }^{p} 0$ terms may be relevant in the application method.

\section{DISCUSSION}

\section{A. Special features of the modified-contracted Schrödinger equations}

As has already been stressed, relations (30), (33), (34), and (35) are self-contained equations. This means that the kind of indeterminacy, which hindered initially the use of the CSE and which in recent years has been approximately removed through the use of construction algorithms for the high-order RDMs, does not formally exist in the MCSEs. However, since the ${ }^{(4 ; 2,2)} C$ and ${ }^{(3 ; 2,1)} C$ matrix elements appearing in the last terms of the MCSEs relations (30) and (34) are not directly modified by the action of the Hamiltonian at each iteration, the question that could be raised is: what kind of information, not contained in the ${ }^{2} \mathcal{M}$ (the 2-CSE), is carried out by the MCSEs? The answer to this question is that the relations linking the lower-order RDMs with the higher-order RDMs are exactly incorporated into the MCSEs structure. In consequence, no extra approximate construction algorithms for the high-order RDMs are needed, as in the 2-CSE case, in order to solve these equations iteratively.

Another special feature of these equations is that the cancellation of high-order correlation terms, which is hidden in the 2-CSE, appears explicitly in the MCSEs.

It should also be noted that the third- and fourth-order correlation matrices are directly related to the third- and fourth-order $G$ matrices $[25,26]$, respectively. Therefore the inequalities imposing that these matrices be positivesemidefinite are auxiliary conditions on the correlation matrices to be considered jointly with the MCSEs.

In our opinion, these features indicate that the information carried by the 4-MCSEs is more complete than that carried by the 2-CSE in spite of the very close relationship between these two families of equations.

\section{B. The iterative procedure}

The general lines of the MCSEs iterative procedure are very similar to the 2 CSE one. Thus, one starts with an initial set of $(p-1)-,(p-2)-, \ldots$, RDMs, a ${ }^{p} C$, and the energy $E$. These initial data should correspond to a realistic zero-order wave function of the eigenstate that is being investigated. This set of zero-order matrices is replaced in the expression of the corresponding MCSE. After symmetrizing the matrix thus obtained, which will be hereafter called ${ }^{p} \tilde{\mathcal{M}}$, its trace is divided by the trace of the corresponding $p$-RDM, $\left(\begin{array}{c}N \\ p\end{array}\right)$, which yields a new $E$. Then the new ${ }^{p} D$ is obtained by dividing ${ }^{p} \tilde{\mathcal{M}}$ by $E$. All the lower-order RDMs and the ${ }^{p} C$ are obtained from the new ${ }^{p} D$ and with this new set of data a new iteration is initiated.

\section{Some significant results}

In order to test these equations the linear $\mathrm{BeH}_{2}$ molecule was calculated using a basis set of 14 Hartree-Fock spin orbitals. The 3- and the 4-MCSE calculating codes have been programed. The 3-MCSE and 4-MCSE codes evaluate relations (30) and (34), respectively.

These codes were tested with the full-configuration interaction and with the Hartree-Fock sets of matrices as input. These calculations showed that:

(1) The 3-MCSE is satisfied by the RDMs corresponding both to the Hartree-Fock and to the full-configuration interaction eigenstates. When initiating the calculation with these matrices the corresponding result does not vary, irrespective of the number of iterations performed, therefore these are fixed points of the iterative process.

(2) The 4-MCSE is satisfied by the RDMs corresponding to the full-configuration-interaction eigenstates, while Hartree-Fock is no longer a fixed point of the equation. It should be stressed that this property is not common to the 2-CSE one. Thus, while the 2-CSE is satisfied by the set of RDMs corresponding to a full-configuration interaction wave function, when a second iteration is attempted-through the use of the approximating algorithms for the high-order $\mathrm{RDMs}$ - the equation is not any more satisfied, which is obviously due to the fact that the algorithms are not exact.

(3) Although some encouraging results have been obtained, on the whole we cannot claim that the procedures developed guaranty convergence. Therefore, this remains an open question.

\section{Questions that must be investigated}

The preliminary results just mentioned indicate that although from a theoretical point of view the MCSEs show that the many-body problem can be exactly transformed into 
a four-body problem, their usefulness cannot be considered established until the convergence of these equations can be achieved or, alternatively, other solution schemes have been devised. The experience gathered after various different trials indicates that there are three main questions that should be further investigated.

\section{N-representability constraints versus convergence}

The main distinction among the different-codes tested is the way in which they incorporate the $N$-representability [28] constraints. Many-different approaches have been tested in order to overcome the apparently restraining effect of these constraints upon the iterative process. Thus, as the iterations proceed the matrices loose their $N$ representability if no constraints are imposed. On the other hand, when the constraints are included, the process advances and then returns to the initial situation. That is, it all happens as if, when forced towards $N$ representability the matrices take the closer $\mathrm{N}$-representable form that happens to be their previous one. The most-successful trial has been one that imposes the $\mathrm{N}$-representability conditions to the 4-, 3-, 2-, and 1-RDM in an independent way without constraining these matrices to be consistent among themselves through contraction.

\section{Should the antisymmetry property be explicitly implemented?}

This question is intimately related to the previous one since the antisymmetry of the RDMs is itself an $N$-representability condition. It is evident that each of the $p$-MCSE forms reported here is just one among the severalpossible forms, since the fermion creator/annihilator operators may be ordered in many-different ways. One can therefore generate an antisymmetrized $p$-MCSE by taking the mean of all these possible forms. In this way one would handle antisymmetrized correlation matrices. Although this feature is very appealing, it lengthens considerably the calculations, that is why we have been reluctant to consider it initially. On the other hand, if the antisymmetry is built in the MCSE equations, the $N$-representability error at each iteration would decrease that could favor convergence. Concerning this issue, let us finally point out that the 4-RDM decomposition and explicit use of the ${ }^{p} C$ can be replaced by the use of our previous $p$-RDM construction algorithm that generates antisymmetrical matrices. The ${ }^{p} \boldsymbol{\Delta}$ error, also called $p$-order cumulant [20], would then play the role of the ${ }^{p} C$. This alternative may have been in the mind of Mazziotti when in 1999 he mentioned to Valdemoro that a fourth-order self-contained equation could be constructed. Probably because the talk took place during a walk, at the time, the idea seemed unfeasible and was discarded. We have not focussed our attention here on this possibility because it is less satisfying from a theoretical point of view (the ${ }^{p} 0$ do not arise) and, as in the antisymmetrical MCSEs case, it might be rather expensive.

\section{Role of the vanishing terms}

As has been seen the relation ${ }^{(4 ; 2,2)} 0=0$ must be satisfied at convergence, thus, in the trials carried out, the vanishing terms have been used as convergence tests. However, since during the iterative procedure, the terms ${ }^{p} 0$ are not equal to zero, they should perhaps be explicitly included. Thus, one may ask whether a procedure based on Eq. (35) in preference to Eq. (34) would prove more easy to control. This approach, which presents several difficulties from the computational point of view, is being investigated at present.

It must, however, be noted that Eq. (35) is not the only relation that may be considered in order to include the vanishing terms explicitly. Thus, another way in which these terms may be taken into account consists, as in Eq. (28), in developing only the rhs of the equation; i.e., by imposing that the equation corresponds exactly to the contraction of the Schrödinger equation and corresponds thus to an eigenstate. This approach implies that the rhs. higher-order vanishing terms are not any longer canceled out by the lhs ones and can therefore only be partly taken into account. This alternative is also being considered.

Although the investigation of these three main questions constitutes a large task it is, in our opinion, worthwhile since apart from the future practical applications it will deepen considerably our understanding of the many-body problem.

\section{ACKNOWLEDGMENTS}

We gratefully thank Professor Luis M. Tel for his useful comments and for suggesting an appropriate name for this new family of equations. We are also indepted to Professor Peter Surjan and Agnes Szabadoz for many useful discussions. C.V. acknowledges the financial support granted to this work by the Ministerio de Ciencia y Tecnología, under Project No. BQU2000-1158 and D.R.A. is grateful to financial support from Agencia Española de Cooperación Internacional (AECI)/Sección Mutis.
[1] P. A. M. Dirac, Proc. Cambridge Philos. Soc. 27, 240 (1931).

[2] K. Husimi, Proc. Phys. Soc. Jpn. 22, 264 (1940).

[3] P. O. Löwdin, Phys. Rev. 97, 1474 (1955).

[4] S. Cho, Sci. Rep. Gumma Univ. 11, 1 (1962).

[5] E. R. Davidson, Reduced Density Matrices in Quantum Chemistry (Academic Press, London, 1976).

[6] C. Valdemoro, in Density Matrices and Density Functionals, Proceedings of the A. J. Coleman Symposium, Kingston, Ontario, 1985, edited by R. Erdahl and V. Smith (Reidel, Dor- drecht, 1987), p 275.

[7] Many-Electron Densities and Reduced Density Matrices, edited by J. Cioslowsky (Kluwer, Dordrecht, 2000).

[8] Reduced Density Matrices: Coulson's Challenge, edited by A. J. Coleman and V. I. Yukalov, (Springer Verlag, New York, 2000).

[9] L. Cohen and C. Frishberg, Phys. Rev. A 13, 927 (1976).

[10] H. Nakatsuji, Phys. Rev. A 14, 41 (1976).

[11] L. Cohen and C. Frishberg, J. Chem. Phys. 65, 4234 (1976). 
[12] J. E. Harriman, Phys. Rev. A 19, 1893 (1979).

[13] F. Colmenero and C. Valdemoro, Int. J. Quantum Chem. 51, 369 (1994).

[14] C. Valdemoro, Phys. Rev. A 45, 4462 (1992).

[15] F. Colmenero, C. Pérez del Valle, and C. Valdemoro, Phys. Rev. A 47, 971 (1993).

[16] H. Nakatsuji and K. Yasuda, Phys. Rev. Lett. 76, 1039 (1996); K. Yasuda and H. Nakatsuji, Phys. Rev. A 56, 2648 (1997).

[17] C. Valdemoro, L. M. Tel, and E. Pérez-Romero, Adv. Quantum Chem. 28, 33 (1997).

[18] C. Valdemoro, M. P. de Lara-Castells, E. Pérez-Romero, and L. M. Tel, Adv. Quantum Chem. 31, 37 (1999).

[19] C. Valdemoro, in Topics in Current Chem, edited by P. Surjan, (Springer-Verlag, Berlin, 1999).
[20] D. A. Mazziotti, Phys. Rev. A 57, 4219 (1998).

[21] D. A. Mazziotti, Chem. Phys. Lett. 289, 419 (1998).

[22] D. A. Mazziotti, Int. J. Quantum Chem. 70, 557 (1998).

[23] D. A. Mazziotti, Phys. Rev. A 60, 3618 (1999).

[24] D. A. Mazziotti, Chem. Phys. Lett. 326, 212 (2000).

[25] C. Valdemoro, L. M. Tel, and E. Pérez-Romero, Phys. Rev. A 61, 032507 (2000).

[26] C. Valdemoro, L. M. Tel, and E. Pérez-Romero, in Manyelectron Densities and Reduced Density Matrices, edited by J. Cioslowsky (Kluwer, Dordrecht, 2000).

[27] L. M. Tel, E. Pérez-Romero, C. Valdemoro, and F. J. Casquero, Int. J. Quantum Chem. 82, 131 (2001).

[28] A. J. Coleman, Rev. Mod. Phys. 45, 4462 (1963). 